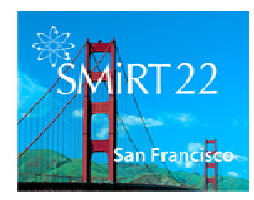

Transactions, SMiRT-22

San Francisco, California, USA - August 18-23, 2013

Division II

\title{
A TIME SAVING METHOD TO COMPUTE MULTI-PASS WELD RESIDUAL STRESSES
}

\section{Frédérique Rossillon ${ }^{1}$ and Lionel Depradeux ${ }^{2}$}

\author{
${ }^{1}$ Dr-Eng, EDF SEPTEN,Villeurbanne, France (frederique.rossillon@edf.fr) \\ ${ }^{2}$ Dr-Eng, EC2-Modélisation, Villeurbanne, France
}

\begin{abstract}
In order to obtain the residual stress field resulting from the welding process, numerical simulation of multipass welding has proved efficient and has become an interesting alternative to practical measurement. However, in the context of engineering studies, it remains a difficult task to compute residual stresses for a very high number of passes with reasonable computation times. In this paper, a time-saving method is proposed to compute the welding process, ensuring an accurate reproduction of the residual stress field with drastically reduced computing times.

The method consists in including in the simulation only the last deposited pass, or a reduced number of appropriately selected passes. For a given material and a given heat input, the choice of remaining passes depends on the geometrical parameters. The method is applied to various geometries of austenitic pipes girth welds, which have been widely studied in the literature and standards. The results, confronted to multi-pass simulations including all the passes, and to literature results, are very satisfactory. Quasi-identical residual stress fields are computed in both cases with computation times divided by a factor comprised between 7 up to 12 . Further computations are in progress on other configurations than girth-weld pipes, and more complex 3D geometry like J welds.
\end{abstract}

\section{INTRODUCTION}

To have a better assessment of the in service loading of PWR component, residual stresses generated by the welding operation are investigated. Since major developments have been achieved in this field, finite element simulation of welding has become a very interesting and straightforward means of prediction of residual stresses generated during welding, and a good alternative to measurements. A large amount of academic or industrial studies considering experimental versus numerical results have demonstrated the relevancy of weld numerical simulations: Smith et al. (2009), Muranski et al. (2011), and Ogawa et al..(2009). However, in the context of engineering studies, it remains a difficult task to compute residual stresses with reasonable computation times. In particular, for thick components such as pressure vessels of PWR's, weld residual stresses are difficult to compute by FE simulation. This is due to the high number of passes involved, and also to the complex geometry of the welded areas. Therefore, full 3D computations are required.

Despite recent progresses in the field of parallel computation, engineering departments still resort to simplified methods to compute weld residual stresses by FE simulations. Among these methods, a common one is the "macro-bead" technique, which consists in merging together two or more passes into one weld block. Therefore, instead of analyzing the thermo-mechanical response for every weld pass, the numerical simulations are performed only for a few “macro-beads". This enables to reduce computation times on 3D geometries (see Watson et al. (2006), Rossillon and Depradeux (2011)). However, this technique, though adequate in some cases, shows some limitations, particularly concerning the determination of the heat inputs that have to be considered in the macro-beads. Such procedure usually requires some fitting of the model data in order to reproduce accurately numerical results or experimental measurements. Moreover, the choice of the macro-bead is often arbitrary.

Recent investigations on multi-pass welding computations on the welded divider plates of steam generators (Rossillon and Depradeux (2011)) showed some promising trails concerning simplified methods of residual stresses computation. Among these, the idea of simulating only the last bead, sometimes considered in early weld numerical simulations (Brickstad and Josefson (1998)), seems very interesting, but is not always suitable for very deep grooves. During our investigations on welded divider plates, it was however observed that, in many cases, the computation of only the last deposited bead, or of a few appropriately selected passes, could be sufficient to ensure a very good reproduction of the stress field. This was observed not only in the vicinity of the last bead, but also in the whole welded area, even in cases for which a large number of passes was involved.

In this paper, we focus only on weld residual stresses in pipe girth welds, for which many well-documented numerical or experimental solutions exist in the open literature. Moreover, the weld residual stress generation 
phenomena in such structures are well understood (Bouchard (2007), Legatt (2008), Dong (2007)). In addition, numerical investigations on such configurations can be performed with 2D axisymetrical models, which require low CPU times. Therefore, the method could be evaluated rather quickly on such geometries, before investigating more complex cases with 3D geometries.

Different numerical test cases have been defined, in order to cover a wide range of configurations, with various numbers of passes. Then a complete multi-pass weld simulation is performed for each test, in order to settle a reference residual stress field. This reference solution is then validated by comparison with existing numericalexperimental solutions from the literature on similar configurations. Afterwards, different simplified simulations are detailed and compared to the reference results, including the simulation of only the last bead instead of the totality. The results are then discussed. Finally, a practical selection procedure of the passes that must be taken into account in the analysis is proposed, based on mechanical and geometrical criteria.

\section{TESTS DEFINITION}

The test cases are typical of pipe girth welds configurations. The study focuses only on austenitic stainless steel pipes, in order to avoid solid-state phase transformation consideration. , In the past two decades, residual stresses in austenitic pipe girth welds have been widely investigated, using either experimental and-or numerical methods, generally toward a goal of fracture mechanics based defect assessment (Stacey et al.(2000)), or on purpose of corrosion investigation. A great amount of results is currently available in the open literature for different weld pipe configurations and for various materials. These results led to compilations of through-wall residual stress distributions such as those given in assessment code procedures (Lee and Chang (2008)). More recently, the development of weld numerical simulations by FE method led to the generalization of parametric studies allowing a deep comprehension of residual stress formation mechanisms in pipes. As a result, synthetic papers as those of Dong (2007) highlighted the parameters (mainly geometrical) governing the residual stress distribution in pipes and vessels girth welds. In parallel, other works, as those of Bouchard (2007), made critical reviews of existing through-wall residual stress distributions, and proposed accurate residual stress profiles for different configurations. These profiles were corroborated by measurements using various methods. It should be pointed out that, despite the good knowledge of residual stresses in pipes, multi-pass welding simulation in pipes are still relevant in the industry at least for two reasons: firstly, some particular restraint conditions may invalidate the recommended residual stress profiles (Legatt (2008)), and numerical simulation is helpful to take into account special boundary conditions; Secondly, some special welding procedures might require a full $3 \mathrm{D}$ analysis to catch the gradient along the circumference of the pipe (Lee and Chan (2008)).

The test cases geometries have been defined so as to cover the entire range of residual stresses distributions in austenitic weld pipes (from "bending type" to "self-equilibrated type” according to Dong (2007), and to remain close enough to well-documented solutions (either experimental, numerical or both) of the open literature. The geometry and heat input parameters are summarized in figure 1 and 3 . The heat input parameters are typical for such kind of configurations.

Table 1: Configuration of the tests

\begin{tabular}{|c|c|c|c|c|}
\hline & Test case 1 & Test case 2 & Test case 3A & Test case 3B \\
\hline & $15 \mathrm{~mm}$ & $20 \mathrm{~mm}$ & \multicolumn{2}{|c|}{$30 \mathrm{~mm}$} \\
\hline Groove geometry & \begin{tabular}{|l|l|l|}
9 & 11 & 10 \\
6 & 8 & 7 \\
4 & 5 \\
2 & 3 \\
& 1
\end{tabular} & 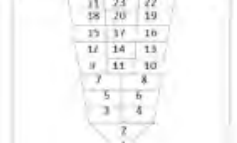 & & \\
\hline Thickness [mm] & 15 & 30 & 60 & 60 \\
\hline Inner radius R [mm] & 150 & 300 & 600 & 6000 \\
\hline $\mathrm{R} / \mathrm{t}$ & 10 & 10 & 10 & 100 \\
\hline Number of beads & 11 & 23 & 45 & 45 \\
\hline $\begin{array}{l}\text { Heat input } \mathrm{E} \\
{[\mathrm{kJ} / \mathrm{mm}]}\end{array}$ & $\begin{array}{c}P_{1}: E=0.7 \\
P_{2} \text { to } P_{11}: E=1.1\end{array}$ & $\begin{array}{c}\mathrm{P}_{1}: \mathrm{E}=0.7 \\
\mathrm{P}_{2}: \mathrm{E}=1.1 \\
\mathrm{P}_{3} \text { to } \mathrm{P}_{23}: \mathrm{E}=1.7\end{array}$ & $\begin{array}{c}\mathrm{P}_{1}: \mathrm{E}=0.7 \\
\mathrm{P}_{2}: \mathrm{E}=1.1 \\
\mathrm{P}_{3} \text { to } \mathrm{P}_{45}: \mathrm{E}=2.5\end{array}$ & $\begin{array}{c}\mathrm{P}_{1}: \mathrm{E}=0.7 \\
\mathrm{P}_{2}: \mathrm{E}=1.1 \\
\mathrm{P}_{3} \text { to } \mathrm{P}_{45}: \mathrm{E}=2.5\end{array}$ \\
\hline
\end{tabular}




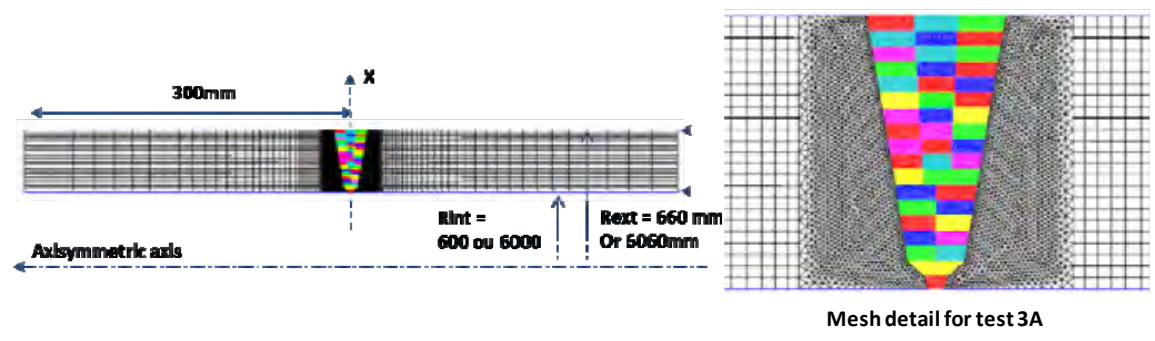

Figure 1: Mesh details for test $3 \mathrm{~A}$ and $\mathrm{B}$

\section{REFERENCE MULTI-PASS COMPUTATION}

For each test, the reference results are obtained by a multi-pass computation using a classical FE welding simulation. The computation is performed with the FE Code Code_Aster ${ }^{\circledR}$ developed by EDF. The mesh and the weld details are shown in fig. 1. Each pass is computed individually and is included in the model gradually according to the weld sequence (see Table 1). At first, the thermal analysis is performed and the time-dependent temperature field is saved for the subsequent mechanical analysis. The transient temperature history is computed by solving the heat conduction equation, assuming temperature-dependent physical properties. The latent heat of fusion is neglected, as it has proven to have negligible effect on the result. The weld passes are included in the model gradually following their deposition.

The heat input is described as an internal volumetric body heat source, prescribed in each bead individually. This body heat source is constant in space over the bead, but vary linearly with time, in order to reproduce the approaching and moving away of the welding torch. This description of the heat input allows calibrating the parameters $\Delta \mathrm{tm}$ and $\varphi$ max for a given prescribed heat input, as follows:

$$
\varphi_{\max } \Delta t_{m} S=E=\eta \frac{u I}{V}
$$

In the Eqn. 1, fixed values of $\Delta \mathrm{t}_{\mathrm{m}}$ dnu ur une ned mpu $\mathrm{e}$ give urrectly the value of $\varphi_{\max }$. Then, knowing the heat input $\mathrm{E}$, there is only one parameter, only $\Delta \mathrm{t}_{\mathrm{m}}$ has to be adjusted. The parameter is then fitted to ensure a maximal temperature equal or exceeding the fusion temperature in all points of the bead. This method has proven its efficiency on other studies, by comparisons with full 3D simulations including more complex moving heat sources. It is now commonly used in EDF studies (Rossillon and Depradeux (2011)).

Radiative and convective heat exchanges with the environment are also taken into account. However, these parameters have a weak effect on the thermal results, as heat conduction in the pieces is the main heat exchange phenomenon. After the thermal transient computation, the mechanical analysis is performed with the thermal history as an input for the thermal strain computation using temperature-dependent material properties. An elasto-plastic model with linear kinematic hardening, and temperature-dependent material properties, is considered, with classical values for austenitic steel.

For simplification purpose, it is assumed that the weld deposit and the base material have the same mechanical properties as the base metal. Despite the yield stress mismatch between the weld and the parent material could possibly affect the results in some ways, it does not change the overall through-thickness residual stress distribution as demonstrated by Dong (2007), and this can be excluded from the analysis. It is also demonstrated that the $\mathrm{t}$ and $\mathrm{R} / \mathrm{t}$ ratio are the critical parameters for the stress distribution.

The reference solutions for the stress field, calculated for each tests, are given on fig. 2:

a) Test 1 exhibits a typical "bending" stress pattern, with an inversion of the sign of the stress through the thickness at the center of the weld, and a "mirror" pattern of the axial stress profiles along the pipe axis between the outer and the inner surfaces. On the contrary, the hoop stresses are more uniform through the thickness, with tensile stresses near the weld, and compressive stresses surrounding the weld area

b) Test 2 presents a less pronounced bending stress pattern, as the hoop restraint is counterbalanced by the axial one. This is due to the large thickness of the pipe. As a result, less tensile stress are generated at the inner surface

c) Test 3A and B exhibit both typical "self-equilibrated” stress patterns, with compressive axial stresses in the middle of the thickness. However, the radius influence is clearly visible on the inner surface of the pipe, for both hoop and axial stresses. 
$22^{\text {nd }}$ Conference on Structural Mechanics in Reactor Technology San Francisco, California, USA - August 18-23, 2013

Division II- ID675
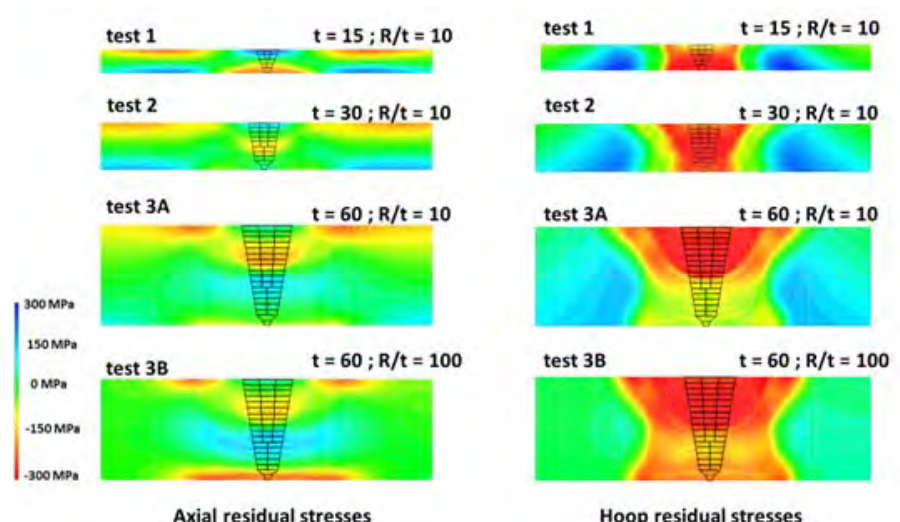

Hoop residual stresses

Figure 2: Reference results obtained with a complete bead by bead analysis for each tests

Test-case 1
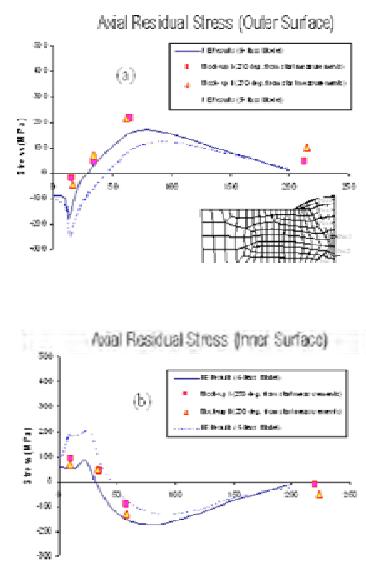

Dong (2007)

$\mathrm{t}=16 ; \mathrm{R} / \mathrm{t}=25 ; \mathrm{X}$ weld

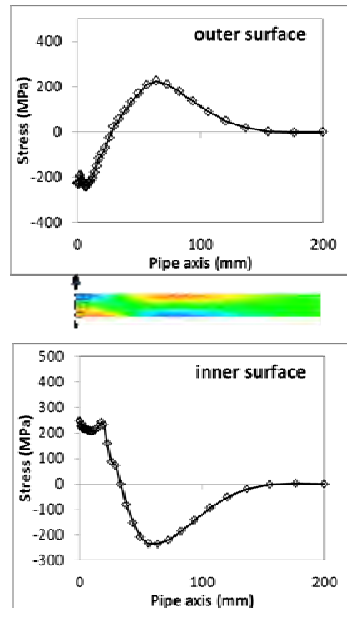

Simulation, $\mathrm{t}=15 ; \mathrm{R} / \mathrm{t}=10$
Test-case $3 A$ and $3 B$

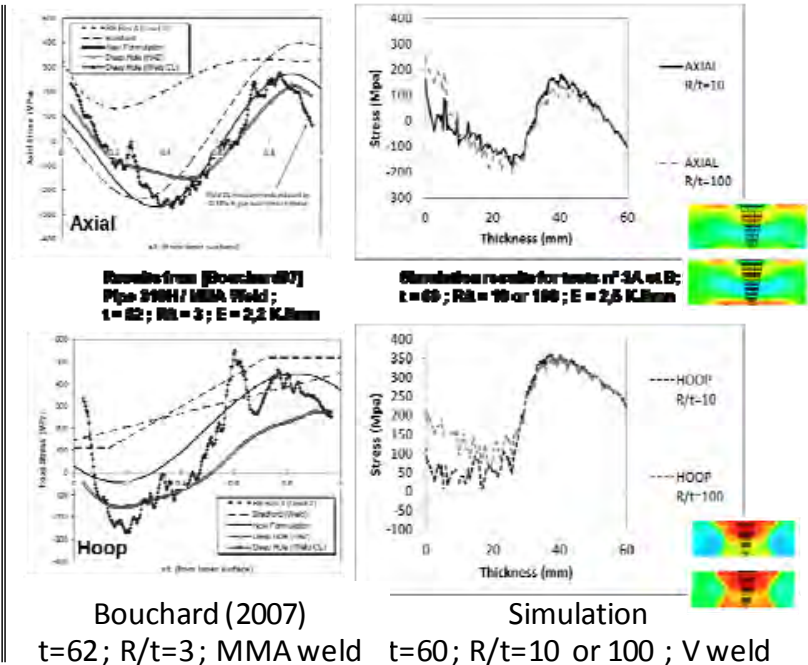

\section{Test-case 2 - axial stress}
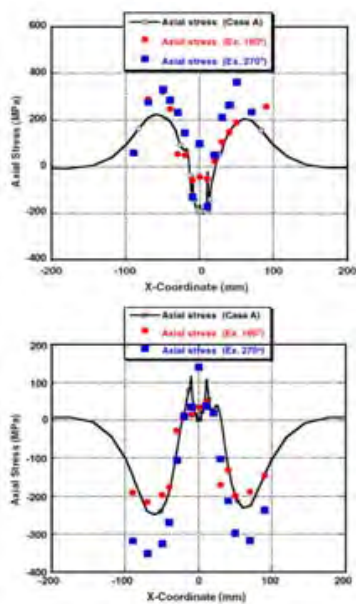

Deng et al.(2008)
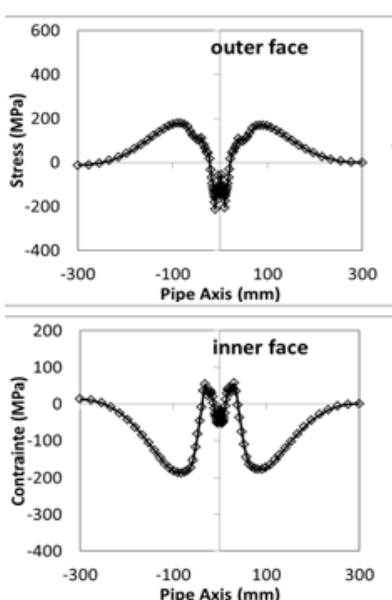

Simulation - $t=30 ; R / t=10$;
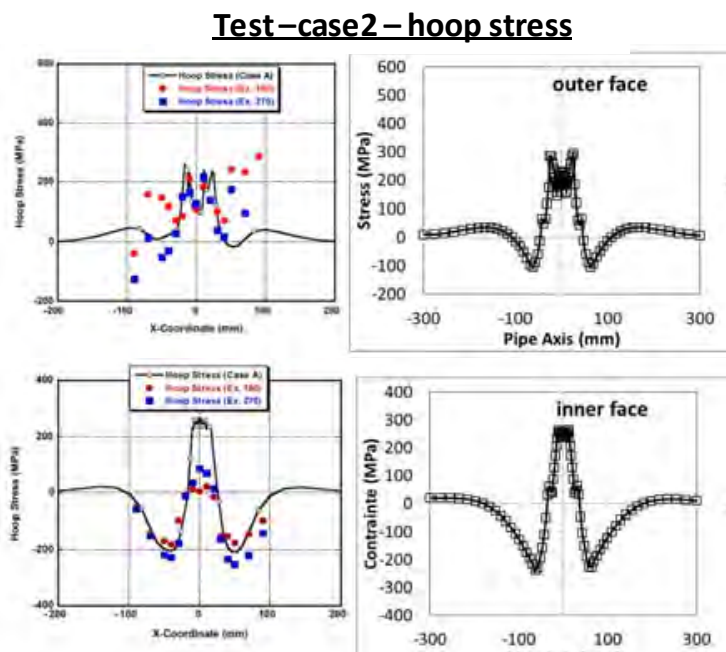

Deng et al.(2008) Simulation - $t=30 ; R / t=10 ;$

Figure 3: Comparison of stress profiles calculated with Code_Aster with results of the literature for test case 1, 2 , 3A and 3B. 
The results have been compared with a panel of literature results on similar configurations (Bouchard (2007), Legatt (2008), Dong (2007) and Deng et al. (2008). It showed a very good agreement, accrediting the relevancy of our computation procedure. As an example,the left-upper side of Figure 3 compares the results for test 1 with different profiles taken from Dong (2007). A typical axial stress pattern is observed for that configuration.

The down part of Figure 3 also compares the results from test 2 with the results from Deng et al. (2008) on a similar configuration (austenitic pipe, 14 beads, $\mathrm{t}=23 \mathrm{~mm}, \mathrm{R}=85 \mathrm{~mm}, \mathrm{E}=1$ to $3 \mathrm{KJ} / \mathrm{mm}$ ). The computed stress profiles show very good agreement with the experimental and numerical results from Deng et al. (2008), although the material data, heat input and geometry are slightly different.

Results of the through-wall stress evolution along the thickness for test 3A and 3B are also compared with results from Bouchard (2007) on fig. 3 (right-upper side). The overall computed evolutions are similar to literature's results on such kind of configuration, though the R/t values are larger on test $3 \mathrm{~A}$ and $3 \mathrm{~B}$ than in the cases considered in Bouchard (2007). Moreover, higher heat input is responsible for a larger zone of stress decrease at the approach of the outer surface, for both tests 3A and 3B. This tendency is confirmed by the available measurements.

\section{SIMULATIONS WITH SIMPLIFIED METHOD}

The principle of the method is to select the last bead, or a small number of appropriately selected beads, and to run the simulation of welding in the same way as for the multi-pass calculation, but only for the retained beads.

For test cases 1 and 2, only the last bead deposit has been considered in the numerical analysis. This means that at the initial state, all the beads except the last one are present in the FE model, but with zero stress or displacement. Only the deposit of the last bead is computed, with exactly the same methodology as for the multi-pass calculation previously presented. In particular, the heat input prescribed in the selected bead is the same as it would have been in the complete multi-pass analysis.

The corresponding results of the simplified analyses for tests 1 and 2 are confronted to the results of the reference multi-pass simulations on figure 4 and 5 . The results are quite interesting: the stress states are almost exactly the same for the simplified methods as they would have been for the complete multi-pass ones. In addition, figure 5 shows that the results for tests 1 and 2 are also very close to literature results, strengthening the robustness of the analyses.

For both tests ( 1 and 2$)$, the simulation of the last bead only is sufficient to reproduce the stress gradients and maximum, not only at the surface of the pipe, but also through the thickness. This is particularly interesting for test 2, as the obtained results with a single bead computation are almost the same as the numerical (and experimental) ones obtained in Deng et al. (2008) with a 14 passes computation.
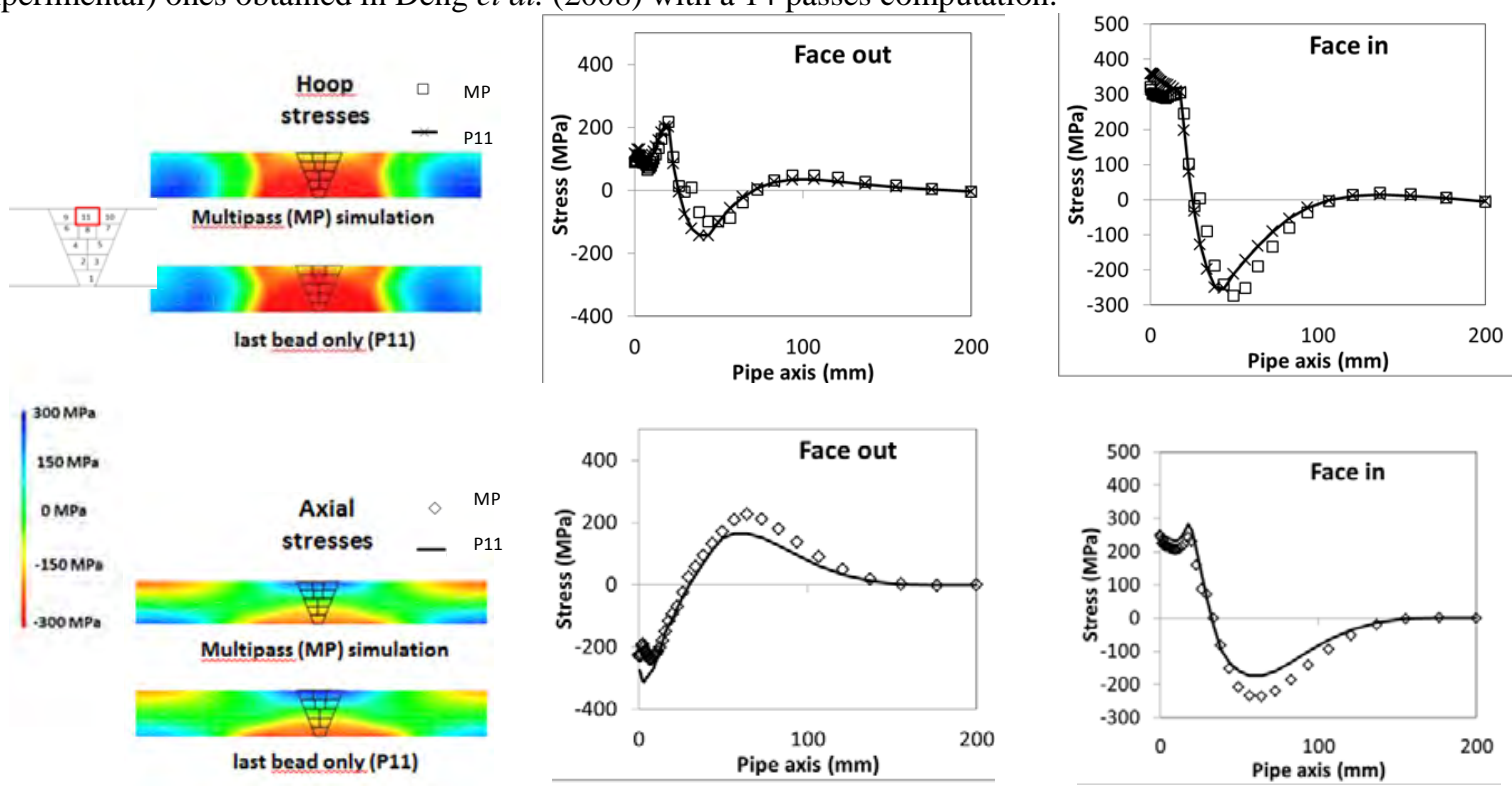

Figure 4: Comparison between the multi-pass simulation (MP) and the simplified method (P11) for test 1 


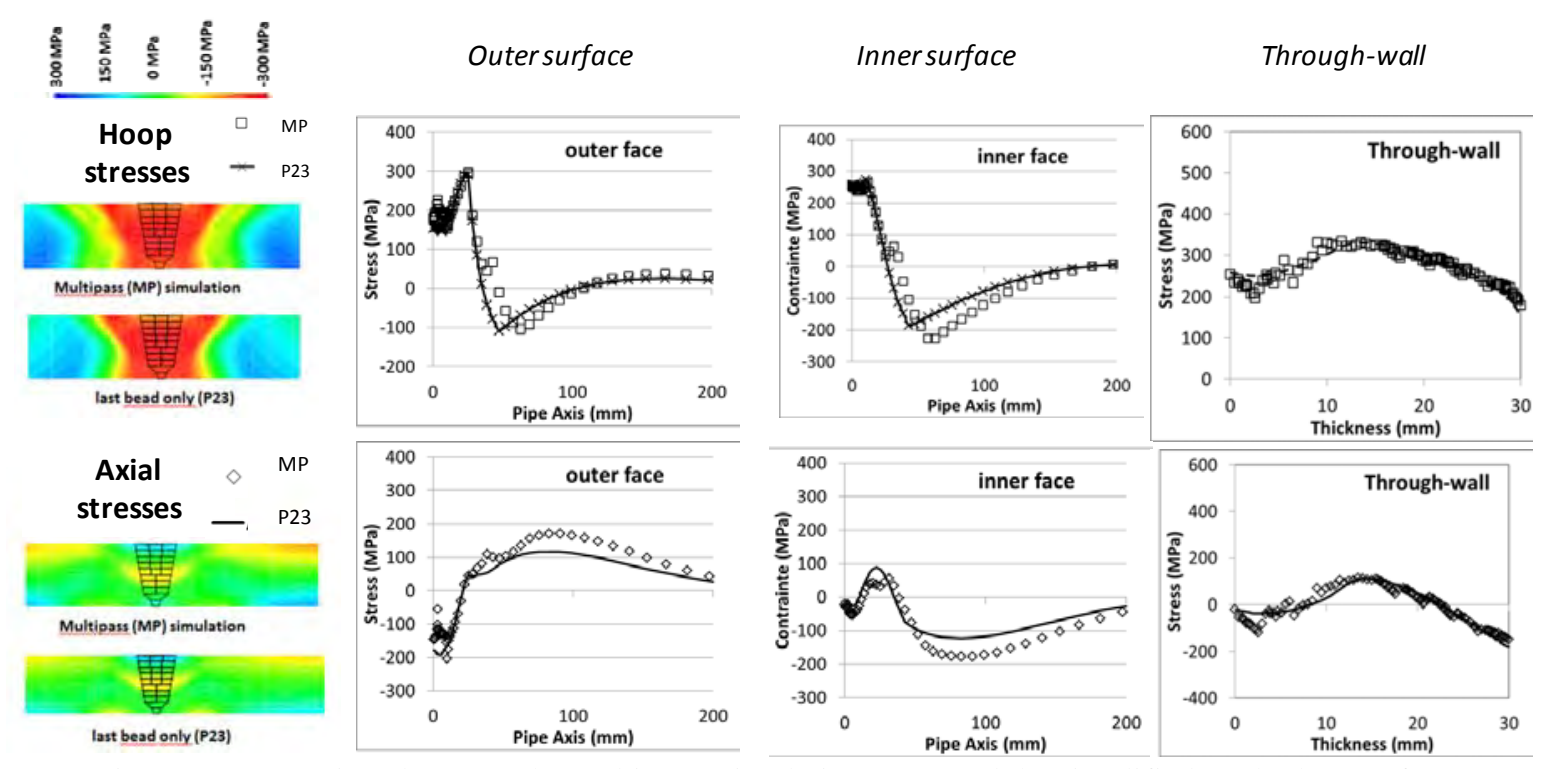

Figure 5: Comparison between the multi-pass simulation (MP) and the simplified method (P23) for test 2

However, as expected, in test cases $3 \mathrm{~A}$ and $3 \mathrm{~B}$ for which the thickness is larger, the simulation of the last deposited bead only, fails to reproduce accurately the stress state obtained by the complete multi-pass simulation. A closer look at the plastic zone shows that there is a close relationship between the spatial distribution of the weld residual stresses in the circumferential direction, and the shape of the plastic zone around the bead (figure 6).

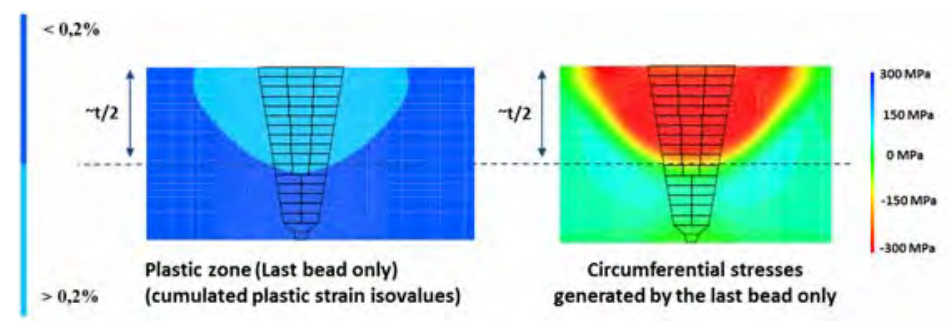

Figure 6: Size of the plastic zone and hoop stresses calculated for the last bead only on test 3A

Following this observation, if one consider only a few intermediate passes in the thickness, it seems possible to cover the plastic zone of the entire weld joint. The idea is to cover the same plastic zone as the one that would be generated by the whole bead by bead simulation. In order to determine which bead will be selected in the simulation, one has to consider the size of the plastic area around each bead. Assuming a cylindrical distribution of the plastic zone around the bead, the radius of the zone can be estimated by the following equation (R6 (2004)):

$$
P Z R=\sqrt{\frac{2 E \alpha}{\sigma_{Y} e \pi \rho C} \frac{Q}{V}}
$$

If the value of PZR is larger than the thickness of the wall, the last deposited layer only can be retained, and the deposit of the other ones may be neglected in the analyses. Moreover, if the last layer contains only a small number of beads, and that the last bead is deposited in the center of the layer (as for the present test cases), only the last bead could be considered. On the contrary, if the last layer is composed of a large number of beads, the beads located at both sides of the layer should be taken into account in order to reproduce accurately the axial stress gradients along the outer surface.

For test cases 1 and 2, the radius of the plastic zone is larger than the thickness of the pipe, and therefore, the last bead is sufficient to compute accurately the stress fields. For test cases $3 \mathrm{~A}$ and $3 \mathrm{~B}$, the last bead is not sufficient, as the corresponding plastic zone does not cover the whole plastic zones associated with the multi-pass simulations. Figure 7 shows some results of parametric studies considering different combinations of selected weld passes. 
$22^{\text {nd }}$ Conference on Structural Mechanics in Reactor Technology San Francisco, California, USA - August 18-23, 2013

Division II- ID675

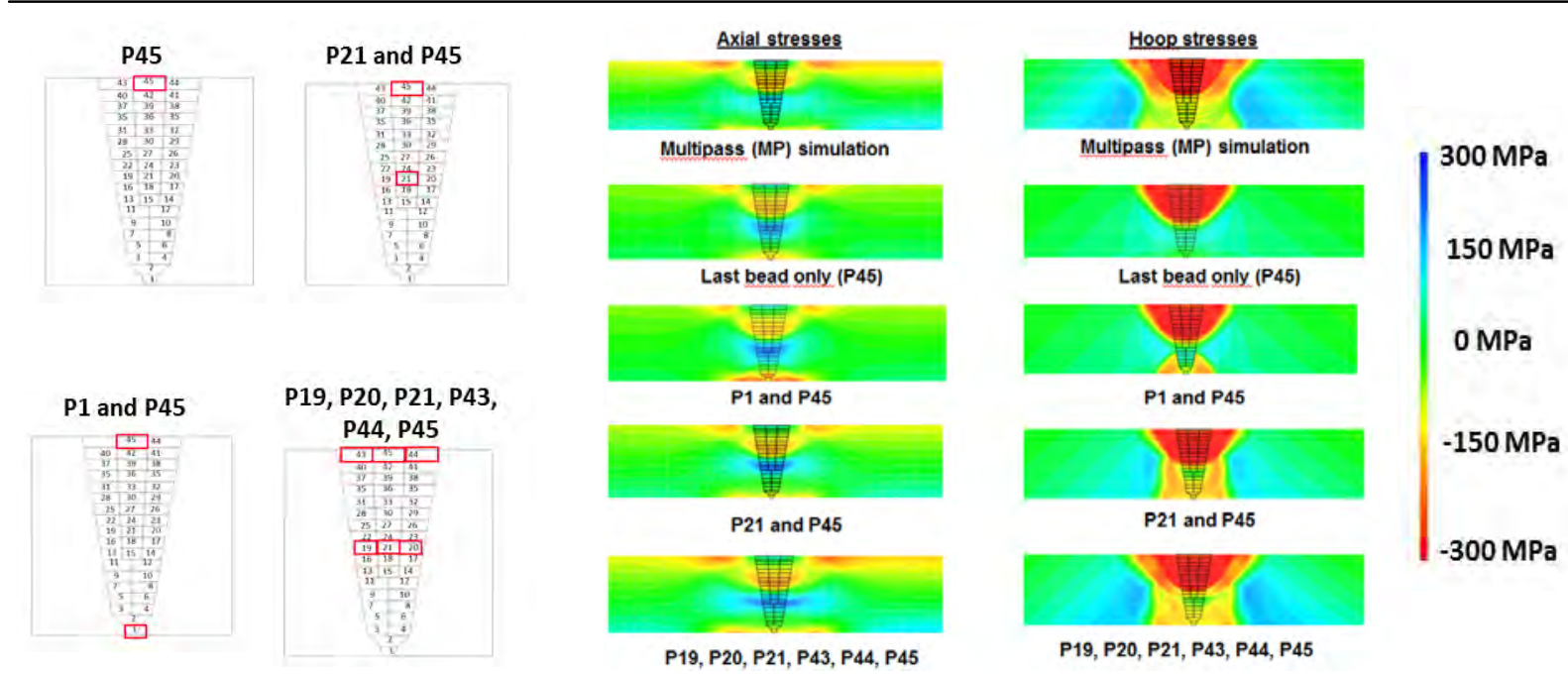

Figure 7: Comparison between the multi-pass simulation (MP) and the different simplified simulations

The optimal choice of the beads that should be considered for test case 3 is given in figure 8 . In that case, the deposit of beads 19, 20 and 21 are simulated, assuming that passes 1 to 18 are already present in the model, but with a zero initial stress field. Following computation of pass 21, passes 22 to 42 are included in the FE model without any stress, and the deposit of beads 43,44 and 45 are finally simulated (thermal and mechanical transient evolutions) from the stress-state resulting from the previous step (end of pass 21). With this method, numerical weld simulations are performed for only 6 passes instead of 43, allowing an noticeable time saving. In this case, the computation time is divided by 7.

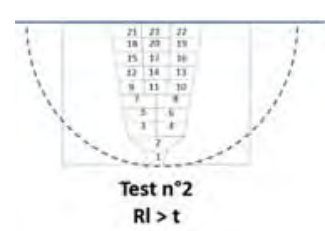

$\mathrm{RI}>\mathrm{t}$
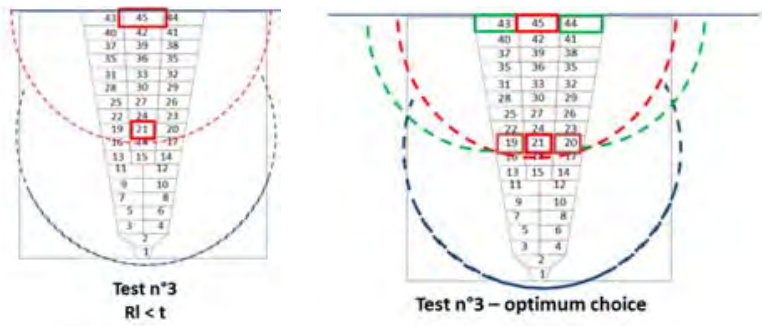

Figure 8: Size of the plastic zone associated with different simplified simulations
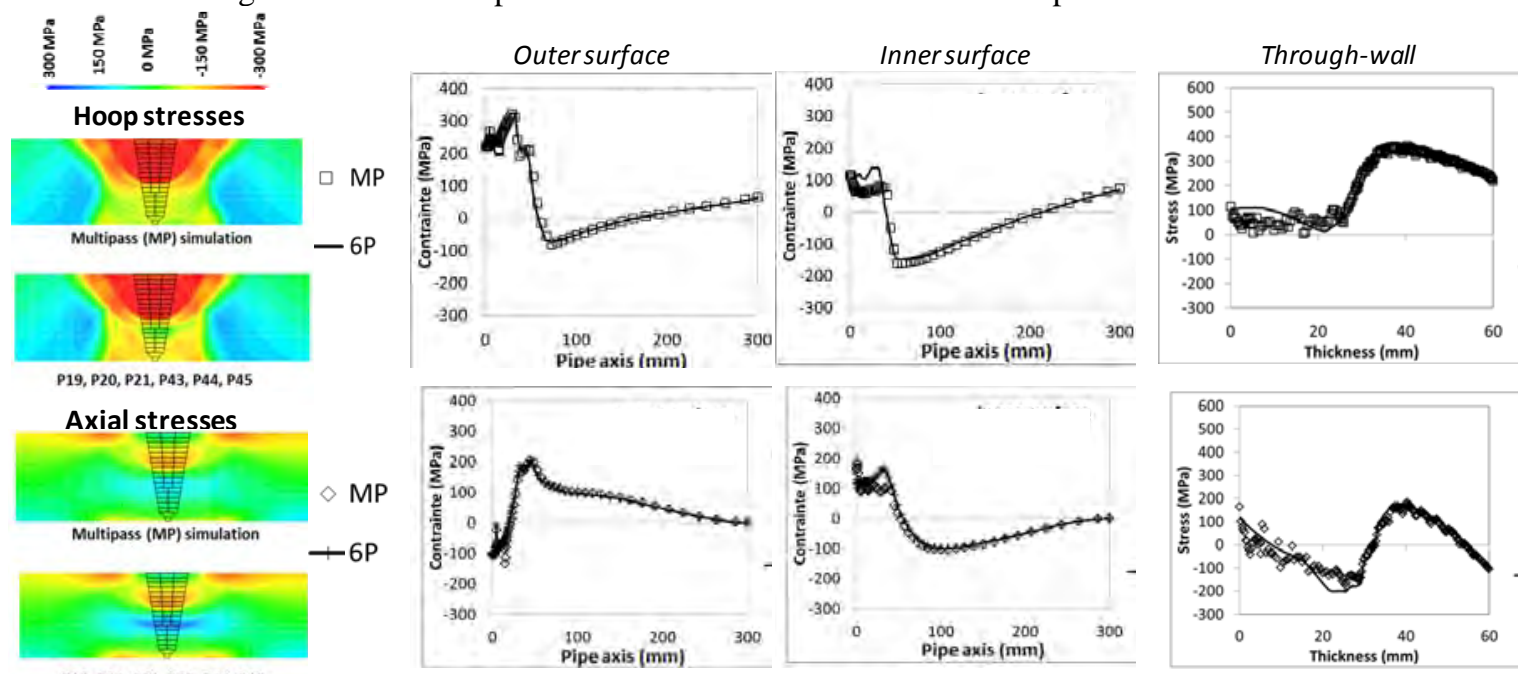

Figure 9: Test 3A -Comparison between the multi-pass simulation (MP) and the simplified simulation 
The final result with this simplified method is compared to the multi-pass one on figure 9 and 10. A very good agreement is obtained with the reference results, for the outer and inner residual stress profiles in both axial and hoop directions, as well as for the through-wall stress evolutions.
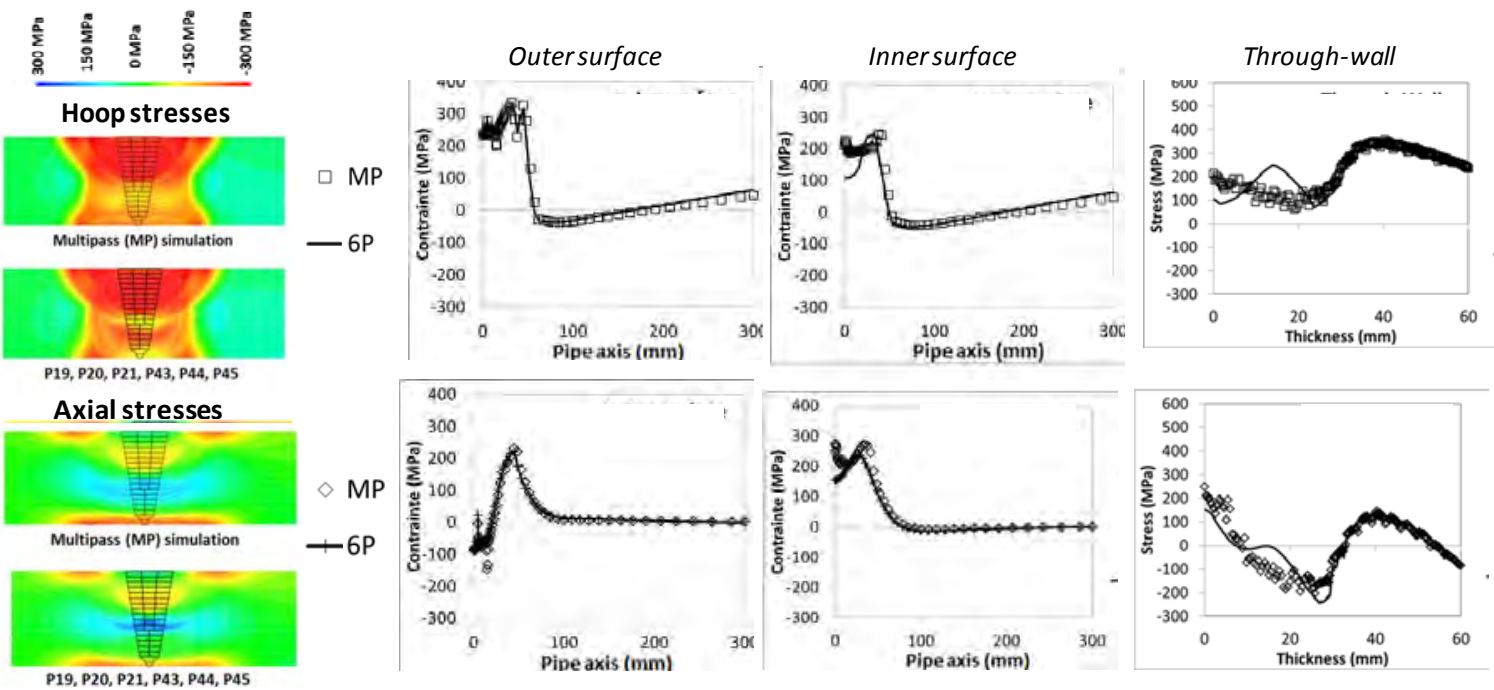

Figure 10: Test 3B Comparison between the multi-pass simulation (MP) and the simplified simulation

To compare the results of the different simulations, the axial and circumferential stress profiles along the outer surface, the inner surface, and through the thickness, have been considered for each test. For each profile, the average discrepancy (absolute value) over the whole profile, between the multi-pass computation and the simplified one (result difference at every calculation point), has been calculated. The correspondent discrepancies, for each profile, are summarized in table 1. Then, the average discrepancy is calculated and compared to the room temperature yield stress of the base metal (table 2). It shows that the average differences, reported to the yield stress, are less than $15 \%$ for all the cases, which is very satisfactory considering the corresponding reduction of time computation.

Table 2 gives a recap of the CPU times required for each tests. The reduction is drastic for all the tests. Though the CPU times are not very high for 2D models even for the complete multi-pass simulations, one has to consider the expected time saving for 3D simulations, for which a single pass can take more than one day of CPU time. The reduction from 43 passes to only 6 passes, as for test 3, would then be particularly attractive. This would make a multi-pass weld FE simulation on very big components within an engineering framework feasible.

Table 2: Average discrepancies in MPa between the multi-pass simulation (MP) and the simplified simulation for test 1 to 3

\begin{tabular}{|l|l|l|l|l|}
\cline { 2 - 5 } \multicolumn{1}{c|}{} & test 1 & test 2 & test 3A & test 3B \\
\hline & P11 only & P23 only & $\begin{array}{l}\text { P19 P20 P21 } \\
\text { P43 P44 P45 }\end{array}$ & P19 P20 P21 \\
\hline Outer surface - Axial stresses & 45 & 39 & 13 & 16 \\
\hline Outer surface - Hoop stresses & 23 & 34 & 11 & 11 \\
\hline Inner surface - Axial stresses & 32 & 31 & 27 & 46 \\
\hline inner surface - Hoop stresses & 43 & 35 & 36 & 60 \\
\hline Through wall - Axial stresses & 26 & 17 & 24 & 33 \\
\hline Through wall - Hoop stresses & 69 & 11 & 21 & 31 \\
\hline Average (all profiles) & $\mathbf{4 0}$ & $\mathbf{2 8}$ & $\mathbf{2 2}$ & $\mathbf{3 3}$ \\
\hline Average / yield stress & $\mathbf{1 4} \%$ & $\mathbf{1 0} \%$ & $\mathbf{8} \%$ & $\mathbf{1 1} \%$ \\
\hline
\end{tabular}

Table 3: Indicative computation time for the different multi-pass and simplified simulations (test 1 to 3 ), for a same computer and a same number of CPU 


\begin{tabular}{|c|c|c|c|}
\hline & Test case 1 & Test case 2 & Test case 3 \\
\hline Multi-pass modeling & $\mathbf{2 5} \mathbf{~ m i n}-11$ beads & $\mathbf{2 h 3 0}-23$ beads & $\mathbf{1 7 h 3 0}-45$ passes \\
\hline Simplified method & $\mathbf{2} \mathbf{~ m i n ~ - 1 ~ b e a d ~}$ & $\mathbf{7} \mathbf{~ m i n}-1$ bead & 2h20 - 6 passes \\
\hline Time ratio & $\mathbf{1 2}$ & $\sim / 20$ & $\sim / 7$ \\
\hline
\end{tabular}

\section{METHODOLOGY RECOMMANDATION}

The successive steps of the simplified method can be summarized as follows:

a) First, estimation of the plastic zone radius (PZR) corresponding to each bead of the groove. On classical configurations such as pipes or plates, this could be achieved using classical analytical formulas such as Eqn.2, knowing the thermal and mechanical parameters and heat input. For more specific geometries, this can be done numerically by running a FE simulation for a single bead, and by analyzing the corresponding PZR

b) Knowing a PZR for each layer, the selection of the retained beads is performed, chronologically from the last deposited bead of the weld sequence, to the first deposited one. Considering the last layer, all the weld passes that are present within the PZR are excluded from the analysis. Then the last deposited layer before the excluded ones is selected, and the radius of its plastic zone is evaluated. Then the selection process is repeated until the very first layer of the weld sequence is reached.

c) In every selected layer, the beads composing the layers are selected according to the same methodology as in b). If the layer contains only a few beads, only the last bead of the layer may be selected. If the layer contains more beads, they must be selected from the last deposited one, going back to the first one, according to the plastic zone's size. As an example, figure 15 shows a specific sequence in a 6 beads layer. Only passes $n^{\circ} 1,2$ and 6 are to be taken into account in the analysis.

d) The simulation of welding of the selected beads is conducted as it is for the complete multi-pass analysis. The unselected beads are also added in the model following the weld sequence, but with zero stress fields, allowing very fast computations.

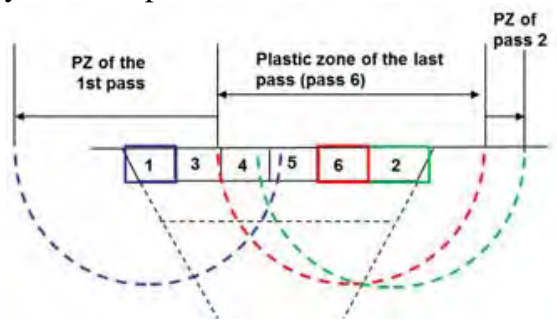

Figure 11: Bead selection in a given layer - only beads 1, 2 and 6 can be taken in the weld analysis

The proposed simplified method is an interesting alternative to macro-bead procedures, even if the computation time is higher (Rossillon and Depradeux (2013) it does not involve any fitting of the input data. It must be emphasized that the modeling of each selected bead deposit is performed in the same manner as in the complete multi-pass analysis. So the heat input in the selected beads can be deduced directly from weld parameters, as defined previously. Therefore, this method is a also a "predictive" one (no fitting). Of course, this simplified method, suitable for weld residual stresses predictions, is not adequate for predicting the amount of cumulated plastic strains in the weld zone.

\section{CONCLUSION}

In this paper, a simplified method to compute weld residual stresses in multi-pass welds with FE method is proposed. This method consists in selecting a few appropriately selected beads, and to simulate their deposit as it is done in a complete multi-pass weld simulation, adding the other bead in the model with a zero stress state. This method allows a drastic reduction of computation times: CPU time are divided by a factor 10 . This is very promising and interesting for 3D models. For the moment, this method has been well validated on different configurations of pipes girth welds, and a practical method to select the beads retained has been defined.

Though very promising, this methodology has to be validated on other configurations than austenitic weld pipes. The application of this method to an industrial component (a steam generator divider plate), is described in 
another paper (Rossillon and Depradeux (2013), and compared to multi-pass results and to a macro beads analysis. In parallel, further investigations of this method are being performed on a full 3D model like $\mathrm{J}$ welds.

\section{ACKNOWLEDGMENTS}

The authors want to acknowledge Dr. Claude Amzallag for introducing the idea of selecting an appropriately reduced number of passes.

\section{NOMENCLATURE}

PWR Pressurised Water Reactor $\quad$ Q/V $\quad$ welding energy per meter units

MP Multi-pass $\quad \alpha \quad$ Thermal coefficient

PZR Plastic Zone Radius $\quad$ oy $\quad$ Yield stress

E Young modulus $\quad \rho \quad$ Density

U Tension $\quad$ S specific heat

I Intensity $\quad t \quad$ Pipe thickness

$\eta \quad$ Efficiency parameter depending on $\quad \mathrm{R} \quad$ Pipe radius

V velocity of the welding torch

S cross section of the pass

\section{REFERENCE}

Bouchard P.J. (2007), "Validated residual stress profiles for fracture assessments of stainless steel pipe girth welds", International Journal of Pressure Vessels and Piping, 84, 195-222.

Brickstad B. and Josefson B. L. (1998), “A parametric study of residual stresses in multi-pass butt-welded stainless steel pipes”, International Journal of Pressure Vessels and Piping, 75, 11-25.

Deng D., Murakawa H. and Liang W. (2008), "Numerical and experimental investigations on welding residual stress in multi-pass butt-welded austenitic stainless steel pipe”, Computational Materials Science 42, 234-244.

Dong P. (2007), “On the Mechanics of Residual Stresses in Girth Welds”, Journal of Pressure Vessel Technology 19, 345-353.

Lee C.H. and Chang K.H. (2008), “Three-dimensional finite element simulation of residual stresses in circumferential welds of steel pipe including pipe diameter effects”, Materials Science and Engineering A 487 210-218

Leggatt R.H. (2008), “Residual stresses in welded structures”, International Journal of Pressure Vessels and Piping, $85,144-151$.

Liu C., Zhang J.X. and Xue C.B. (2011), "Numerical investigation on residual stress distribution and evolution during multi-pass narrow gap welding of thick-walled stainless steel pipes”, Fusion Engineering and Design 86, 288-295.

Muránsky O., Smith M.C., Bendeich P.J. and Edwards L. (2011), "Validated numerical analysis of residual stresses in Safety Relief Valve (SRV) nozzle mock-ups”, Computational Materials Science 50, 2203-2215.

Ogawa K., Deng D, Kiyoshima S., Yanagida N. and Saito K. (2009) "Investigations on welding residual stresses in penetration nozzles by means of 3D thermal elastic plastic FEM and experiment”, Computational Materials Science, 45, 1031-1042.

R6 - Assessment of the integrity of structures containing defects, British Energy, 2004

Rossillon F. and Depradeux L. (2011), “A methodology for a simplified thermo-mechanical welding simulation in an engineering framework”, Proc. SMiRT 21, India Div-III ID 407

Rossillon F. and Depradeux L. (2013), "Simplified computation of the welding process on a steam-generator divider plate “,PVP2013-97238, ASME 2013 Pressure Vessels and Piping Conference, Paris, France

Stacey A., Barthelemy J.-Y., Leggatt R.H. and Ainsworth R.A. (2000), "Incorporation of residual stresses into the SINTAP defect assessment procedure”, Engineering Fracture Mechanics 67 573-611.

Smith M.C. and Smith A., C. (2009), "NeT Bead-on-plate round robin: Comparison of transient thermal predictions and measurements”, International Journal of Pressure Vessels and Piping 86, 96-109

Watson C. T., Gregg A., Dennis R., Leggatt N., Kingston E. and Smith D.J. (2006), "Residual Stress Finite Element Analysis and Measurements of a Tube Penetration J-Groove Attachment Weld in a Hemispherical Head of a Large Ferritic Pressure Vessel - Part I Centre Nozzle”, Proc.PVP2006-ICPVT-11-2006, ASME PVP, Vancouver, Canada 